\title{
Numerical Solution of Acoustic Scattering by an Adaptive DtN Finite Element Method
}

\author{
Xue Jiang ${ }^{1}$, Peijun $\mathrm{Li}^{2}{ }^{2 *}$ and Weiying Zheng $^{3}$ \\ ${ }^{1}$ LSEC, Institute of Computational Mathematics, Academy of Mathematics and \\ Systems Science, Chinese Academy of Sciences, Beijing, 100190, China. \\ 2 Department of Mathematics, Purdue University, West Lafayette, Indiana, 47907, \\ $U S A$. \\ ${ }^{3}$ LSEC, Institute of Computational Mathematics, Academy of Mathematics and \\ System Sciences, Chinese Academy of Sciences, Beijing, 100190, China.
}

Received 30 October 2011; Accepted (in revised version) 27 April 2012

Communicated by Gang Bao

Available online 8 October 2012

\begin{abstract}
Consider the acoustic wave scattering by an impenetrable obstacle in two dimensions, where the wave propagation is governed by the Helmholtz equation. The scattering problem is modeled as a boundary value problem over a bounded domain. Based on the Dirichlet-to-Neumann (DtN) operator, a transparent boundary condition is introduced on an artificial circular boundary enclosing the obstacle. An adaptive finite element based on a posterior error estimate is presented to solve the boundary value problem with a nonlocal DtN boundary condition. Numerical experiments are included to compare with the perfectly matched layer (PML) method to illustrate the competitive behavior of the proposed adaptive method.
\end{abstract}

AMS subject classifications: 65M30, 78A45, 35Q60

Key words: Helmholtz equation, DtN boundary condition, adaptive finite element method, a posteriori error estimate.

\section{Introduction}

We propose and study an adaptive finite element method with the Dirichlet-to-Neumann $(\mathrm{DtN})$ boundary condition for solving the acoustic wave scattering by a bounded and

${ }^{*}$ Corresponding author. Email addresses: jxue@lsec.cc.ac.cn (X. Jiang), lipeijun@math.purdue.edu (P. Li), zwy@lsec.cc.ac.cn (W. Zheng) 
impenetrable obstacle. The acoustic wave propagation can be modeled by the twodimensional Helmholtz equation:

$$
\begin{aligned}
& \Delta u+k^{2} u=-f, \quad \text { in } \mathbb{R}^{2} \backslash \bar{D}, \\
& u=g, \quad \text { on } \Gamma_{D}, \\
& \lim _{r=|x| \rightarrow \infty} \sqrt{r}\left(\frac{\partial u}{\partial r}-\mathbf{i} k u\right)=0
\end{aligned}
$$

where the wavenumber $k>0$ is assumed to be a positive constant, $D$ is the bounded obstacle with Lipschitz continuous boundary $\Gamma_{D}, f \in L_{\text {loc }}^{2}\left(\mathbb{R}^{2} \backslash \bar{D}\right)$ and $g \in H^{1 / 2}\left(\Gamma_{D}\right)$. The boundary condition $(1.1 \mathrm{~b})$ is not essential. The results can be easily extended to solve the obstacle scattering problem with other boundary conditions such as Neumann or impedance boundary condition on $\Gamma_{D}$, or to solve the acoustic wave propagation through inhomogeneous media with a variable wavenumber inside some bounded domain.

The obstacle scattering problem has played a fundamental role in diverse scientific areas such as radar and sonar, geophysical exploration, non-destructive testing, and medical imaging [7]. Due to its significant applications, this problem has been extensively investigated by a variety of computational methods such as finite difference method, finite element method [16,19] including adaptivity [13,14], and boundary integral method [6] including improved formulations to eliminate the spurious resonances [17]. In order to apply the finite element method, the unbounded physical domain needs to be truncated into a bounded computational domain. Therefore, suitable boundary conditions are needed to imposed on the truncated domain so no artificial wave reflection occurs there $[8,10,11,21]$. Recently, an adaptive perfectly matched layer (PML) techniques has been proposed by $\mathrm{Chen}$ and $\mathrm{Wu}$ [4] for solving the wave propagation by periodic structures. The basic idea of the PML technique is to surround the computational domain with a finite thickness of layer of specially designed model medium, which would either slow down or attenuate all the waves that propagate from inside the computational domain. The adaptive PML technique was extended by Chen and Liu [5] to solve the obstacle scattering problem afterwards. The present work is concerned with an alternative adaptive finite element method that uses the transparent boundary condition instead of the PML technique. The transparent boundary condition is based on the DtN operator and is exact, and thus the artificial boundary can be put as close as possible to the obstacle. Numerical examples will be presented and compared with the adaptive PML technique in [5] in terms of the convergence rate, condition numbers, and a posterior error estimates. We refer to [12] for a related work on the error analysis of the DtN finite element method for solving the acoustic scattering problem via Fourier analysis. We hope the idea developed in this work will be useful for solving other scattering problems on unbounded domains, and even broader scientific problems posed on unbounded domains where the PML techniques might not be applicable. We refer to Bao et al. [3] and Li [18] for an adaptive finite element method with DtN boundary condition to solve a related diffraction grating problem. 
The paper is organized as follows. In Section 2, we reformulate the unbounded domain problem into a bounded domain problem using the DtN operator. For the truncated bounded domain problem, finite element approximations are studied in Section 3. Section 4 is devoted to a posterior error estimate for the adaptive finite element method. In Section 5, we describe our adaptive algorithm and present several numerical examples to compare with the PML method and to demonstrate the competitive behavior of the proposed adaptive method.

\section{Well-posedness of the weak problem}

We start by introducing some notation and Sobolev spaces used in this paper. Let $L^{2}(\Omega)$ be the usual Hilbert space of square integrable functions equipped with the following inner product and norm:

$$
(w, v):=\int_{\Omega} w(x) \bar{v}(x) \mathrm{d} x \quad \text { and } \quad\|w\|_{L^{2}(\Omega)}:=(w, w)^{1 / 2},
$$

where $\bar{v}$ denotes the complex conjugate of $v$. Throughout the paper we will use the usual $H^{1}$-norm over a bounded domain $\Omega \subset \mathbb{R}^{2}$ :

$$
\|w\|_{1, \Omega}=\left(\|w\|_{L^{2}(\Omega)}^{2}+\|\nabla w\|_{L^{2}(\Omega)}^{2}\right)^{1 / 2},
$$

where $|\Omega|$ is the area of the domain $\Omega$.

Let $B_{R}=\left\{x \in \mathbb{R}^{2}:|x|<R\right\}$ be the ball with radius $R>0$ and centering at the origin and let $\Gamma=\partial B_{R}$ be its boundary. For convenience, we assume $\bar{D} \subset B_{R}$ and define $\Omega=B_{R} \backslash \bar{D}$. In the exterior domain $\mathbb{R}^{2} \backslash \bar{B}_{R}$, the solution of the Helmholtz equation can be written in polar coordinates as follows [6]:

$$
u(r, \theta)=\sum_{n \in \mathbb{Z}} \frac{H_{n}^{(1)}(k r)}{H_{n}^{(1)}(k R)} \hat{u}_{n} e^{\mathrm{i} n \theta}, \quad \hat{u}_{n}=\frac{1}{2 \pi} \int_{0}^{2 \pi} u(R, \theta) e^{-\mathbf{i} n \theta} d \theta, \quad \text { for all } r>R,
$$

where $H_{n}^{(1)}(\cdot)$ is the Hankel function of the first kind with order $n$. In this section, we shall establish the weak solution of (1.1) in $\Omega$ by introducing the Dirichlet-to-Neumann $(\mathrm{DtN})$ operator on the truncation boundary $\Gamma$.

For any function $v \in L^{2}(\Gamma)$ with the Fourier expansion $v=\sum_{n \in \mathbb{Z}} \hat{v}_{n} e^{\mathbf{i} n \theta}$, we define an equivalent $L^{2}(\Gamma)$ norm of $v$ by using the Fourier coefficients:

$$
\|v\|_{0, \Gamma}=\left(2 \pi \sum_{n \in \mathbb{Z}}\left|\hat{v}_{n}\right|^{2}\right)^{1 / 2}
$$

The trace space of functions in $H^{1}\left(B_{R}\right)$ is defined by

$$
H^{1 / 2}(\Gamma)=\left\{v \in L^{2}(\Gamma):\|v\|_{\frac{1}{2}, \Gamma}<\infty\right\}
$$


with the norm characterized by

$$
\|v\|_{\frac{1}{2}, \Gamma}^{2}=2 \pi \sum_{n \in \mathbb{Z}}\left(1+n^{2}\right)^{1 / 2}\left|\hat{v}_{n}\right|^{2} .
$$

We shall also use the space $H^{-1 / 2}(\Gamma)$ which is the dual space of $H^{1 / 2}(\Gamma)$, i.e., $H^{-1 / 2}(\Gamma)=$ $\left(H^{1 / 2}(\Gamma)\right)^{\prime}$. The norm in $H^{-1 / 2}(\Gamma)$ is given by

$$
\|v\|_{-\frac{1}{2}, \Gamma}^{2}=2 \pi \sum_{n \in \mathbb{Z}}\left(1+n^{2}\right)^{-1 / 2}\left|\hat{v}_{n}\right|^{2}
$$

Now we introduce the DtN operator $\mathcal{T}: H^{1 / 2}(\Gamma) \rightarrow H^{-1 / 2}(\Gamma)$ as follows

$$
\mathcal{T} v=\frac{1}{R} \sum_{n \in \mathbb{Z}} h_{n}(k R) \hat{v}_{n} e^{\mathrm{i} n \theta}, \quad \text { for all } v \in H^{1 / 2}(\Gamma),
$$

where

$$
h_{n}(z):=z \frac{H_{n}^{(1)^{\prime}}(z)}{H_{n}^{(1)}(z)} \quad \text { and } \quad \hat{v}_{n}=\frac{1}{2 \pi} \int_{0}^{2 \pi} v e^{-\mathbf{i} n \theta} \mathrm{d} \theta .
$$

Following [20], we can write $h_{n}(z)$ into

$$
h_{n}(z)=-\frac{f_{n}(z)}{g_{n}(z)}+\mathbf{i} \frac{z}{g_{n}(z)}
$$

where

$$
f_{n}(z)=\sum_{m=0}^{n}(m+1) c_{n}^{m} z^{-2 m}, \quad g_{n}(z)=\sum_{m=0}^{n} c_{n}^{m} z^{-2 m}, \quad c_{n}^{m}=\frac{(m+n) !(2 m) !}{4^{m}(m !)^{2}(n-m) !} .
$$

Evidently, we have

$$
1 \leq-\operatorname{Re} h_{n}(z) \leq n+1, \quad 0<\operatorname{Im} h_{n}(z) \leq z, \quad \text { for all } z>0 .
$$

The DtN operator admits the following properties.

Lemma 2.1. There exists a constant $C>0$ depending on $k$ and $R$ such that, for any $0 \neq w \in$ $H^{1 / 2}(\Gamma)$,

$$
\|\mathcal{T} w\|_{-\frac{1}{2}, \Gamma} \leq C\|w\|_{\frac{1}{2}, \Gamma^{\prime}}, \quad-\operatorname{Re}\langle\mathcal{T} w, w\rangle \geq C\|w\|_{0, \Gamma}^{2}, \quad \operatorname{Im}\langle\mathcal{T} w, w\rangle>0,
$$

where $\langle\cdot, \cdot\rangle$ stands for the duality pairing between $H^{-1 / 2}(\Gamma)$ and $H^{1 / 2}(\Gamma)$ or the inner product on $L^{2}(\Gamma)$. 
Proof. By (2.5) and the norms on $H^{-1 / 2}(\Gamma)$ and $H^{1 / 2}(\Gamma)$, we have

$$
\|\mathcal{T} w\|_{-\frac{1}{2}, \Gamma}^{2}=\frac{2 \pi}{R^{2}} \sum_{n \in \mathbb{Z}} \frac{\left|h_{n}(k R)\right|^{2}}{\left(1+n^{2}\right)^{1 / 2}}\left|\hat{w}_{n}\right|^{2} \leq C \sum_{n \in \mathbb{Z}}\left(1+n^{2}\right)^{1 / 2}\left|\hat{w}_{n}\right|^{2}=C\|w\|_{\frac{1}{2}, \Gamma^{\prime}}^{2}
$$

where the constant $C$ only depends on $k$ and $R$. Using the inner product and the Fourier expansion, we arrive at

$$
\langle\mathcal{T} w, w\rangle=\frac{2 \pi}{R} \sum_{n \in \mathbb{Z}} h_{n}(k R)\left|\hat{w}_{n}\right|^{2} .
$$

Thus (2.5) indicates that

$$
\begin{aligned}
& \operatorname{Re}\langle\mathcal{T} w, w\rangle=\frac{2 \pi}{R} \sum_{n \in \mathbb{Z}} \operatorname{Re} h_{n}(k r)\left|\hat{w}_{n}\right|^{2} \leq-\frac{2 \pi}{R} \sum_{n \in \mathbb{Z}}\left|\hat{w}_{n}\right|^{2}=-C\|w\|_{0, \Gamma}^{2}, \\
& \operatorname{Im}\langle\mathcal{T} w, w\rangle=\frac{2 \pi}{R} \sum_{n \in \mathbb{Z}} \operatorname{Im} h_{n}(k r)\left|\hat{w}_{n}\right|^{2}>0 .
\end{aligned}
$$

This completes the proof.

From (2.2) and [7] we know that $\mathcal{T}$ is well-defined and the scattering solution $u$ satisfies

$$
\partial_{n} u=\mathcal{T} u, \quad \text { on } \Gamma,
$$

where $\boldsymbol{n}$ is the unit outward normal to $\Gamma$. We define

$$
H_{\Gamma_{D}}^{1}(\Omega):=\left\{v \in H^{1}(\Omega): v=0 \text { on } \Gamma_{D}\right\} .
$$

Then a weak formulation of (1.1) reads: Find $u \in H^{1}(\Omega)$ such that $u=g$ on $\Gamma_{D}$ and

$$
a(u, v)=(f, v), \quad \text { for all } v \in H_{\Gamma_{D}}^{1}(\Omega),
$$

where the sesquilinear form $a: H^{1}(\Omega) \times H^{1}(\Omega) \rightarrow \mathbb{C}$ is defined by

$$
a(u, v)=(\nabla u, \nabla v)-k^{2}(u, v)-\langle\mathcal{T} u, v\rangle .
$$

Theorem 2.1. The weak problem (2.8) has at most one solution.

Proof. Assume $u_{1}$ and $u_{2}$ are two solutions of (2.8). Then the difference $u:=u_{1}-u_{2} \in$ $H_{\Gamma_{D}}^{1}(\Omega)$ and satisfies

$$
\int_{\Omega}\left(|\nabla u|^{2}-k^{2}|u|^{2}\right) d x-\langle\mathcal{T} u, u\rangle=0
$$

The imaginary part yields

$$
\operatorname{Im}\langle\mathcal{T} u, u\rangle=\frac{2 \pi}{R} \sum_{n \in \mathbb{Z}} \operatorname{Im}\left(h_{n}(k R)\right)\left|\hat{u}_{n}\right|^{2}=0,
$$

which gives $\hat{u}_{n}=0$ for $n \in \mathbb{Z}$ by (2.5). Thus we have $u=0$ and $\partial_{n} u=0$ on $\Gamma$. By the Holmgren uniqueness theorem, we have $u=0$ in $\mathbb{R}^{2} \backslash \Omega$. A unique continuation result [15] concludes that $u=0$ in $\Omega$. 
Theorem 2.2. The variational problem (2.8) admits a unique weak solution $u$ in $H^{1}(\Omega)$. Furthermore, there is a constant $C$ depending on $k$ and $R$ such that

$$
\|u\|_{1, \Omega} \leq C\left(\|f\|_{0, \Omega}+\|g\|_{\frac{1}{2}, \Gamma_{D}}\right) .
$$

Proof. By trace theorem (cf., Adams [1], Theorem 7.53), there exist $u_{0} \in H^{1}(\Omega)$ such that $u_{0}=g$ on $\Gamma_{D}$ and

$$
\left\|u_{0}\right\|_{1, \Omega} \leq C\|g\|_{\frac{1}{2}, \Gamma_{D}}
$$

where $C$ is a constant. In fact, $u_{0}$ is the weak solution of the following problem

$$
\begin{array}{ll}
-\Delta u_{0}+u_{0}=0, & \text { in } \Omega, \\
u_{0}=g, & \text { on } \Gamma_{D}, \\
u_{0}=0, & \text { on } \Gamma .
\end{array}
$$

Consider the variational problem: Find $u \in H^{1}(\Omega)$ such that $u-u_{0} \in H_{\Gamma_{D}}^{1}(\Omega)$ and

$$
a\left(u-u_{0}, v\right)=(f, v)-a\left(u_{0}, v\right), \quad \text { for all } v \in H_{\Gamma_{D}}^{1}(\Omega),
$$

which is equivalent to the following variational problem: Find $w \in H_{\Gamma_{D}}^{1}(\Omega)$ such that

$$
a(w, v)=(h, v), \quad \text { for all } v \in H_{\Gamma_{D}}^{1}(\Omega),
$$

where the linear functional

$$
(h, v)=(f, v)-a\left(u_{0}, v\right) .
$$

We split $a(\cdot, \cdot)=a_{+}(\cdot, \cdot)-k^{2}(\cdot, \cdot)$, where $a_{+}(w, v)=(\nabla w, \nabla v)-\langle\mathcal{T} w, v\rangle$. From Lemma 2.1 we conclude that $a_{+}$is coercive from

$$
\left|a_{+}(v, v)\right| \geq\|\nabla v\|_{0, \Omega}^{2}+|\operatorname{Re}\langle\mathcal{T} v, v\rangle| \geq\|\nabla v\|_{0, \Omega}^{2}+C\|v\|_{0, \Gamma}^{2}=\|\nabla v\|_{0, \Omega}^{2}+C\|v\|_{0, \partial \Omega}^{2},
$$

which implies that there exists a constant $C$ only depending on $k$ and $R$ such that

$$
\left|a_{+}(v, v)\right| \geq C\|v\|_{1, \Omega}^{2}, \quad \text { for all } v \in H_{\Gamma_{D}}^{1}(\Omega) .
$$

Let $\mathcal{K}: L^{2}(\Omega) \rightarrow H^{1}(\Omega)$ be a linear operator defined by

$$
a_{+}(\mathcal{K} w, v)=(w, v), \quad \text { for all } v \in H_{\Gamma_{D}}^{1}(\Omega),
$$

which gives

$$
(\nabla \mathcal{K} w, \nabla v)-\langle\mathcal{T} \mathcal{K} w, v\rangle=(w, v), \quad \text { for all } v \in H_{\Gamma_{D}}^{1}(\Omega) .
$$

The Lax-Milgram lemma and the coercivity of $a_{+}$indicate that $\mathcal{K}$ is well-defined and bounded from $L^{2}(\Omega)$ to $H^{1}(\Omega)$ :

$$
\|\mathcal{K} w\|_{1, \Omega} \leq C\|w\|_{0, \Omega} .
$$


Clearly (2.11) is equivalent to the operator equation

$$
\left(\mathcal{I}-k^{2} \mathcal{K}\right) w=\mathcal{K} h
$$

Since $H^{1}(\Omega)$ is compactly embedded into $L^{2}(\Omega), \mathcal{K}$ is a compact operator. It follows from the uniqueness of the solution and the Fredholm alternative that the operator $\mathcal{I}-k^{2} \mathcal{K}$ has a bounded inverse. We then have the estimate that

$$
\|w\|_{0, \Omega} \leq C\|\mathcal{K} h\|_{0, \Omega} \leq C\|h\|_{0, \Omega} .
$$

From (2.13)-(2.15), we deduce that

$$
\|w\|_{1, \Omega}=\left\|\mathcal{K}\left(k^{2} w+h\right)\right\|_{1, \Omega} \leq C\left\|k^{2} w+h\right\|_{0, \Omega} \leq C\|h\|_{0, \Omega}
$$

which yields

$$
\|u\|_{1, \Omega} \leq\|w\|_{1, \Omega}+\left\|u_{0}\right\|_{1, \Omega} \leq C\left(\|h\|_{0, \Omega}+\left\|u_{0}\right\|_{1, \Omega}\right) .
$$

It follows from the linear functional (2.12) and the trace theorem that

$$
\|u\|_{1, \Omega} \leq C\left(\|f\|_{0, \Omega}+\|g\|_{\frac{1}{2}, \Gamma_{D}}\right)
$$

which completes the proof.

By the general theory in Babuška and Aziz [2, Chap.5], for any $f \in L^{2}(\Omega)$ and $g \in$ $H^{1 / 2}\left(\Gamma_{D}\right)$, the uniqueness and existence of the solution imply the following inf-sup condition

$$
\sup _{0 \neq \psi \in H_{\Gamma_{D}}^{1}(\Omega)} \frac{|a(\varphi, \psi)|}{\|\psi\|_{1, \Omega}} \geq \mu\|\varphi\|_{1, \Omega,} \quad \text { for all } \varphi \in H_{\Gamma_{D}}^{1}(\Omega),
$$

where $\mu$ is a positive constant only depending on $k$ and $R$.

\section{Isoparametric finite element approximation}

Let $\mathcal{M}_{h}$ be a shape regular triangulation of $\Omega$ in the sense that

$$
\max _{T \in \mathcal{M}_{h}}\left(h_{T} / \rho_{T}\right) \leq C,
$$

where $C>0$ is a constant independent of $\mathcal{M}_{h}, h_{T}$ is the diameter of $T$, and $\rho_{T}$ is the diameter of the inscribed ball of $T$. We assume that the intersection $\bar{T}_{1} \cap \bar{T}_{2}$ of any two elements $T_{1}, T_{2} \in \mathcal{M}_{h}$ is either empty or a vertex or an edge. For simplicity, we assume that $\Gamma_{D}$ is piecewise quadratic and all its critical points are nodes of $\mathcal{M}_{h}$. To study isoparametric finite elements, we assume that any edge $E$ is a subset of $\partial \Omega$ if it has two boundary vertices. Thus we have $\bar{\Omega}=\cup_{T \in \mathcal{M}_{h}} \bar{T}$. 
Let $\hat{T}$ be the reference triangle with vertices $(1,0),(0,1)$, and $(0,0)$ and denote $P_{k}$ be the space of all polynomials of order $k$. For any $T \in \mathcal{M}_{h}$, the isoparametric mapping $F_{T}$ : $\hat{T} \rightarrow T$ is defined by

$$
x=F_{T}(\hat{x})=\sum_{i=1}^{3} \hat{\lambda}_{i}\left(2 \hat{\lambda}_{i}-1\right) a_{i}+\sum_{1 \leq i<j \leq 3} 4 \hat{\lambda}_{i} \hat{\lambda}_{j} a_{i j}, \quad \text { for all } \hat{x} \in \hat{T},
$$

where $a_{1}, a_{2}, a_{3}$ are the three vertices of $T, a_{i j}$ is the midpoint of the edge of $T$ whose endpoints are $a_{i}$ and $a_{j}$, and $\hat{\lambda}_{1}=\hat{x}_{1}, \hat{\lambda}_{2}=\hat{x}_{2}, \hat{\lambda}_{3}=1-\hat{x}_{1}-\hat{x}_{2}$ are barycentric coordinates of $\hat{T}$. It is easy to verify that $F_{T}$ is affine if all edges of $T$ are straight. Now we define the piecewise quadratic and isoparametric finite element subspace

$$
V_{h}:=\left\{v \in H^{1}(\Omega):\left(\left.v\right|_{T}\right) \circ F_{T} \in P_{2}(\hat{T})\right\} .
$$

In the weak formulation (2.8), the capacity operator $\mathcal{T}$ given by (2.3) is defined by an infinite series which is unrealistic in the computation. Given a sufficiently large $N$, we define a truncated capacity operator

$$
\mathcal{T}_{N} v=\frac{1}{R} \sum_{|n| \leq N} h_{n}(k R) \hat{v}_{n} e^{\mathrm{i} n \theta}, \quad \text { for all } v \in H^{1 / 2}(\Gamma) .
$$

Let $g_{h}$ be the finite element approximation of $g$ satisfying

$$
g_{h} \in\left\{\left.v_{h}\right|_{\Gamma_{D}}: v_{h} \in V_{h}\right\}
$$

We now have the finite element approximation to (2.8): Find $u_{h}^{N} \in V_{h}$ such that $u_{h}^{N}=g_{h}$ on $\Gamma_{D}$ and

$$
a_{N}\left(u_{h}^{N}, v_{h}\right)=\left(f, v_{h}\right), \quad \text { for all } v_{h} \in V_{h} \cap H_{\Gamma_{D}}^{1}(\Omega),
$$

where the sesquilinear form $a_{N}: H^{1}(\Omega) \times H^{1}(\Omega) \rightarrow \mathbb{C}$ is defined as follows:

$$
a_{N}(u, v)=(\nabla u, \nabla v)-k^{2}(u, v)-\left\langle\mathcal{T}_{N} u, v\right\rangle .
$$

Define $W_{N}:=\operatorname{Span}\left\{e^{\mathbf{i} n \theta}:|n| \leq N\right\}$ which is a subspace of $L^{2}(\Gamma)$ and let $P_{N}: L^{2}(\Gamma) \rightarrow W_{N}$ be the projection operator given by

$$
P_{N} v=\sum_{|n| \leq N} \hat{v}_{n} e^{\mathrm{i} n \theta}, \quad \hat{v}_{n}=\frac{1}{2 \pi} \int_{0}^{2 \pi} v e^{-\mathbf{i} n \theta} d \theta
$$

Then using similar arguments as in [12, Section 5], we can prove the following wellposedness and error estimates of the discrete solution. We do not elaborate on the details here. 
Theorem 3.1. There exist constants $h_{0}>0$ and $N_{0} \geq 0$ such that for any $0<h \leq h_{0}$ and $N \geq N_{0}$, the discrete problem (3.4) has a unique solution. Moreover, if the exact solution $u \in H^{m}(\Omega)$ for some integer $m \geq 2$, then

$$
\left\|u-u_{h}^{N}\right\|_{1, \Omega} \leq C\left\{h^{m-1}+\frac{\left\|u-P_{N} u\right\|_{H^{m-1 / 2}(\Gamma)}}{\|u\|_{H^{m-1 / 2}(\Gamma)}} N^{1-m}\right\}\|u\|_{m, \Omega},
$$

where $h=\max _{T \in \mathcal{M}_{h}} h_{T}$ and $C>0$ is a constant independent of $N$ and $h$.

\section{Residual-based a posteriori error estimates}

The purpose of this section is to deduce the a posterior error estimate for the discrete solution. For any $T \in \mathcal{M}_{h}$, we define the interior residual on $T$ by

$$
R_{T}=f+\mathcal{L}\left(\left.u_{h}^{N}\right|_{T}\right),
$$

where the operator $\mathcal{L}=\Delta+k^{2}$. Let $\mathcal{B}_{h}$ denote the set of all edges of $\mathcal{M}_{h}$. For any $E \in \mathcal{B}_{h}$, we define the jump residual across $E$ as follows:

$$
J_{E}=\left(\left.\nabla u_{h}^{N}\right|_{T_{1}}-\left.\nabla u_{h}^{N}\right|_{T_{2}}\right) \cdot \boldsymbol{n}, \quad \text { on } E=\partial T_{1} \cap \partial T_{2},
$$

where $\boldsymbol{n}$ is the unit normal on $E$ and $T_{1}, T_{2} \in \mathcal{M}_{h}$, and

$$
J_{E}=\sqrt{2}\left(\mathcal{T}_{N} u_{h}^{N}-\nabla u_{h}^{N} \cdot \boldsymbol{n}\right), \quad \text { for all } E \subset \Gamma .
$$

For any $T \in \mathcal{M}_{h}$, the local error estimator $\eta_{T}$ is defined by

$$
\eta_{T}^{2}=\left\|h_{T} R_{T}\right\|_{0, T}^{2}+\frac{1}{2} \sum_{E \subset \partial T} h_{E}\left\|J_{E}\right\|_{0, E}^{2}
$$

where $h_{E}$ is the length for the edge $E$.

The following theorem is the main result of this section.

Theorem 4.1. Let $u$ and $u_{h}^{N}$ be solutions of (1.1) and (3.4) respectively. Then there exist two positive constants $C_{1}, C_{2}$ such that $C_{1}$ only depends on the shape-regularity of $\mathcal{M}_{h}, C_{2}$ only depends on $R, k$, and

$$
\left\|u-u_{h}^{N}\right\|_{1, \Omega} \leq C_{1}\left(\eta_{h}+\left\|g-g_{h}\right\|_{H^{1 / 2}\left(\Gamma_{D}\right)}\right)+C_{2} \eta_{N},
$$

where the a posteriori error estimates are defined by

$$
\begin{aligned}
\eta_{h} & :=\left(\sum_{T \in \mathcal{M}_{h}} \eta_{T}^{2}\right)^{1 / 2}, \\
\eta_{N} & :=\left(\sum_{|n|>N}\left(1+n^{2}\right)^{-1 / 2}\left|h_{n}(k R) \hat{u}_{h, n}^{N}\right|^{2}\right)^{1 / 2}, \quad \hat{u}_{h, n}^{N}=\frac{1}{2 \pi} \int_{0}^{2 \pi} u_{h, n}^{N} e^{-\mathbf{i} n \theta} d \theta .
\end{aligned}
$$


In the above a posteriori error estimates, $\eta_{h}$ characterizes the local errors from finite element discretization, and $\eta_{N}$ corresponds to the truncation error of the approximate DtN operator. The proof of Theorem 4.1 will be postponed to the end of this section.

Lemma 4.1. Let $C_{2}$ be the constant in Theorem 4.1 and $N$ be large enough. Then

$$
\left|\int_{\Gamma}\left(\mathcal{T}-\mathcal{T}_{N}\right) u_{h}^{N} \bar{v} d s\right| \leq C_{2} \eta_{N}\|v\|_{1, \Omega}, \quad \text { for all } v \in H^{1}(\Omega) .
$$

Proof. By the definitions of $\mathcal{T}, \mathcal{T}_{N}$ and the Fourier expansions of $u_{h}^{N}, v$, we have

$$
\begin{aligned}
\left|\int_{\Gamma}\left(\mathcal{T}-\mathcal{T}_{N}\right) u_{h}^{N} \bar{v} d s\right| & =\frac{1}{R}\left|\sum_{|n|>N} h_{n}(k R) \hat{u}_{h, n}^{N} \sum_{m \in \mathbb{Z}} \overline{\hat{v}}_{m} \int_{\Gamma} e^{\mathrm{i}(n-m) \theta} d s\right| \\
& =\left|\sum_{|n|>N} h_{n}(k R) \hat{u}_{h, n}^{N} \overline{\hat{v}}_{n}\right| \leq \eta_{N}\|v\|_{\frac{1}{2}, \Gamma} \leq C_{2} \eta_{N}\|v\|_{1, \Omega} .
\end{aligned}
$$

where we have used the trace theorem in the last inequality.

Lemma 4.2. Let $u, u_{h}^{N}$ be solutions of (2.8) and (3.4) respectively. Then

$$
\left|a\left(u-u_{h}^{N}, v\right)\right| \leq\left(C_{1} \eta_{h}+C_{2} \eta_{N}\right)\|v\|_{1, \Omega}, \quad \text { for all } v \in H^{1}(\Omega) .
$$

Proof. From (2.8) and (3.4) we have, for any $v \in H_{\Gamma_{D}}^{1}(\Omega)$ and $v_{h} \in V_{h} \cap H_{\Gamma_{D}}^{1}(\Omega)$,

$$
\begin{aligned}
a\left(u-u_{h}^{N}, v\right) & =a\left(u-u_{h}^{N}, v-v_{h}\right)+\left\langle\left(\mathcal{T}-\mathcal{T}_{N}\right) u_{h}^{N}, v_{h}\right\rangle \\
& =\left(f, v-v_{h}\right)-a\left(u_{h}^{N}, v-v_{h}\right)+\left\langle\left(\mathcal{T}-\mathcal{T}_{N}\right) u_{h}^{N}, v_{h}\right\rangle \\
& =\left(f, v-v_{h}\right)-a_{N}\left(u_{h}^{N}, v-v_{h}\right)+\left\langle\left(\mathcal{T}-\mathcal{T}_{N}\right) u_{h}^{N}, v\right\rangle .
\end{aligned}
$$

For convenience we let $\rho\left(v-v_{h}\right):=\left(f, v-v_{h}\right)-a_{N}\left(u_{h}^{N}, v-v_{h}\right)$. Then the formula of integration by parts yields

$$
\rho\left(v-v_{h}\right)=\sum_{T \in \mathcal{M}_{h}}\left\{\int_{T} R_{T}\left(\bar{v}-\bar{v}_{h}\right) d x+\frac{1}{2} \sum_{E \subset \partial T \backslash \Gamma_{D}} \int_{E} J_{E}\left(\bar{v}-\bar{v}_{h}\right) d s\right\},
$$

where $R_{T}$ and $J_{E}$ are defined in (4.1)-(4.3).

Now we introduce the Scott-Zhang interpolation operator $\Pi_{h}: H^{1}(\Omega) \rightarrow V_{h}$, which admits the following estimates

$$
\left\|v-\Pi_{h} v\right\|_{0, T} \leq C h_{T}\|\nabla v\|_{0, \widetilde{T}} \quad \text { and } \quad\left\|v-\Pi_{h} v\right\|_{0, E} \leq C h_{E}^{1 / 2}\|\nabla v\|_{0, \widetilde{E}^{\prime}}
$$

where $\widetilde{T}$ and $\widetilde{E}$ are the union of all elements in $\mathcal{M}_{h}$ which share a vertex with $T$ and $E$ respectively. Then taking $v_{h}=\pi_{h} v$ and using the Cauchy-Schwartz inequality, we have

$$
\begin{aligned}
\left|\rho\left(v-\pi_{h} v\right)\right| & \leq \sum_{T \in \mathcal{M}_{h}}\left\{\left\|R_{T}\right\|_{0, T}\left\|v-\Pi_{h} v\right\|_{0, T}+\frac{1}{2} \sum_{E \subset \partial T \backslash \Gamma_{D}}\left\|J_{E}\right\|_{0, E}\left\|v-\Pi_{h} v\right\|_{0, E}\right\} \\
& \leq C \eta_{h}\|v\|_{1, \Omega} .
\end{aligned}
$$

Together with Lemma 4.1 we complete the proof. 
Now we are in the position of proving Theorem 4.1.

Proof of Theorem 4.1. From the trace theorem, there exists a $u_{g h} \in H^{1}(\Omega)$ such that $u_{g h}=0$ on $\Gamma, u_{g h}=g-g_{h}$ on $\Gamma_{D}$, and

$$
\left\|u_{g h}\right\|_{1, \Omega} \leq C\left\|g-g_{h}\right\|_{H^{1 / 2}\left(\Gamma_{D}\right)} .
$$

Then $u-u_{h}^{N}-u_{g h} \in H_{\Gamma_{D}}^{1}(\Omega)$. From the inf-sup condition (2.16) we deduce that

$$
\begin{aligned}
\left\|u-u_{h}^{N}\right\|_{1, \Omega} & =\left\|u_{g h}\right\|_{1, \Omega}+\sup _{0 \neq v \in H_{\Gamma_{D}}^{1}(\Omega)} \frac{\left|a\left(u-u_{h}^{N}-u_{g h}, v\right)\right|}{\|v\|_{1, \Omega}} \\
& \leq C\left\|u_{g h}\right\|_{1, \Omega}+\sup _{0 \neq v \in H_{\Gamma_{D}}^{1}(\Omega)} \frac{\left|a\left(u-u_{h}^{N}, v\right)\right|}{\|v\|_{1, \Omega}} .
\end{aligned}
$$

The proof is completed upon inserting (4.7) and (4.8) into the above inequality.

\section{Adaptive algorithm and numerical experiments}

In this section, we propose an adaptive finite element algorithm with truncated DtN boundary conditions. We shall demonstrate the efficiency of the algorithm by two numerical experiments and compare the results with those obtained by the adaptive PML algorithm. The implementation of our algorithm is based on Matlab.

\subsection{Adaptive algorithms}

First we define the a posteriori error indicators for refining the mesh by

$$
\bar{\eta}_{T}= \begin{cases}\left(\eta_{T}^{2}+h_{E}^{-1}\left\|g-g_{h}\right\|_{0, E}^{2}\right)^{1 / 2}, & \text { if } \partial T \cap \Gamma_{D}=E \text { is an edge, } \\ \eta_{T}, & \text { else, }\end{cases}
$$

for all $T \in \mathcal{M}_{h}$, and define the total a posteriori error estimate by

$$
\bar{\eta}_{h}=\left(\sum_{T \in \mathcal{M}_{h}} \bar{\eta}_{T}^{2}\right)^{1 / 2}
$$

In Theorem 4.1, the a posteriori error contains $\left\|g-g_{h}\right\|_{H^{1 / 2}\left(\Gamma_{D}\right)}$, which is not computable. We choose a computable and good approximation $h_{E}^{-1}\left\|g-g_{h}\right\|_{0, E}^{2}$ in the implementation. In practice, we may simply choose $N$ based on Theorem 4.1 which will be large enough to ensure that the truncation error does not contaminate quasi-optimal behavior of the a posterior error estimate. It could be shown numerically that $N$ does not have to be very 
large and the accuracy of the numerical results is not significantly affected by the truncation of the DtN operator. We may set a criterion to guarantee that $\eta_{N}$ always subordinate to $\bar{\eta}_{h}$ by requiring $\eta_{N} / \bar{\eta}_{h} \leq \tau$ for some small factor $0<\tau \ll 1$.

Now we propose the adaptive algorithm for solving (3.4).

Algorithm 5.1. Given the tolerance $\varepsilon>0, \theta \in(0,1)$, and $N_{0} \geq 0, N_{\text {step }}>0$.

- Fix the computational domain $\Omega=B_{R} \backslash \bar{D}$ by choosing the radius $R$.

- Construct an initial triangulation $\mathcal{M}_{h}$ over $\Omega$ and initialize $N$ to $N_{0}$.

- While $\bar{\eta}_{h}>\varepsilon$ do

1. solve the discrete problem (3.4) on $\mathcal{M}_{h}$;

2. compute the local error indicators $\bar{\eta}_{T}$ for all $T \in \mathcal{M}_{h}$ and the total a posteriori error estimates $\bar{\eta}_{h}, \eta_{N}$;

3. while $\eta_{N} / \bar{\eta}_{h}>\tau$ do

- set $N=N+N_{\text {step }}$;

- repeat step 1 and step 2;

4. refine the mesh $\mathcal{M}_{h}$ according to the following strategy:

refine all elements in $\widehat{\mathcal{M}}_{h}$ which is the smallest subset satisfying

$$
\eta_{\widehat{\mathcal{M}}_{h}}>\theta \bar{\eta}_{h}
$$

end while.

\subsection{Numerical experiments}

We report two numerical experiments based on the linear Lagrange finite element method. The examples are computed by both the adaptive DtN algorithm and the adaptive PML method in [5]. We set the wave number by $k=1, k=\pi, k=2 \pi$ and set $\theta=0.5, \tau=0.005$ in Algorithm 5.1. The complex coordinate stretching of the PML method is defined by

$$
\widetilde{x}=x(1+\mathbf{i} \sigma(r-R)), \quad \text { for all } x \in \mathbb{R}^{2} \backslash \bar{B}_{R},
$$

where $r=|x|$ and $\sigma$ is a positive constant. In our cases, $\sigma$ is so chosen that $k \sigma=10 \pi$.

Example 5.1. Let the scatterer $\mathrm{D}$ be the unit ball and $\Omega=B(0,2)$. In (1.1), the righthand side $f$ and the boundary condition $g$ are chosen such that the exact solution is $u(x)=$ $H_{0}^{(1)}(k|x|)$. We set $N_{0}=3$ and $N_{\text {step }}=1$ in Algorithm 5.1. For the adaptive PML method, the thickness of the PML is set by $\rho=2$ so that the error of PML approximation is negligible.

Fig. 1 shows that the quasi-optimality

$$
\left\|u-u_{h}\right\|_{1, \Omega} \sim C N_{h}^{-1 / 2}, \quad \bar{\eta}_{h} \sim C N_{h}^{-1 / 2} .
$$



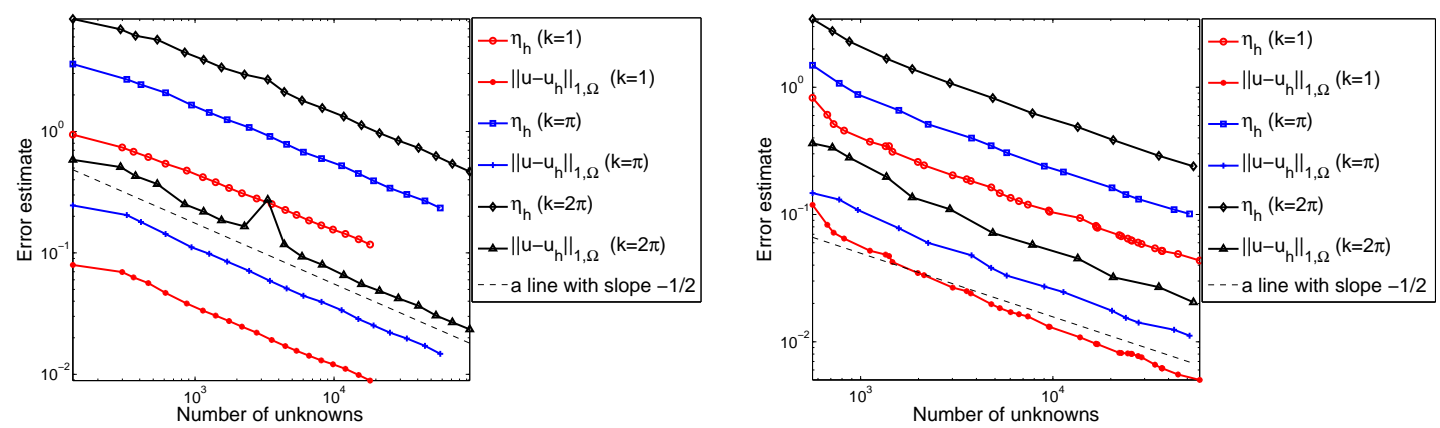

Figure 1: Quasi-optimality of the a priori and a posteriori error estimates for Example 5.1. Left: the adaptive DtN method; Right: the adaptive PML method.
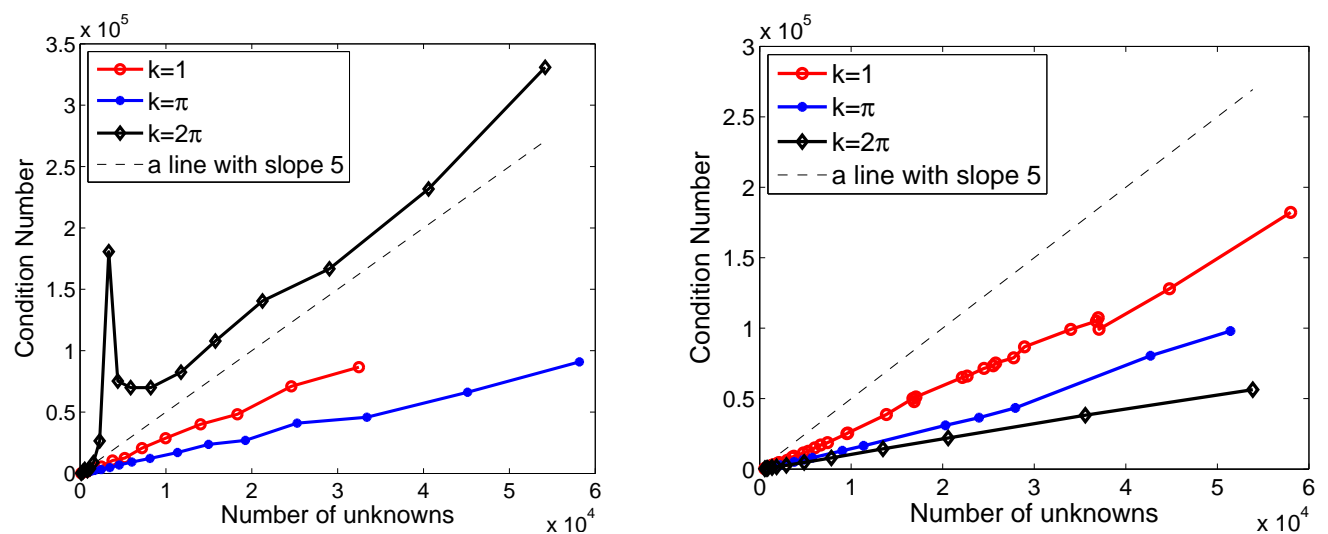

Figure 2: Condition number of the stiffness matrix for Example 5.1. Left: the adaptive DtN method; Right: the adaptive PML method.

hold asymptotically for both the adaptive DtN method and the adaptive PML method for $k=1, \pi, 2 \pi$. Fig. 2 shows the condition numbers of the finite element stiffness matrices for the adaptive DtN method and the adaptive PML method respectively. They are comparable in terms of approximation errors and condition numbers (except for the case of $k=2 \pi$ in Fig. 2).

Figs. 3-8 show the adaptively refined meshes and the plots of the discrete solution for different $k$. In fact, the adaptive DtN algorithm yields $N=3$ for $k=1, \pi$ and $N=5$ for $k=2 \pi$. In this case, the time for computing $\mathcal{T}_{N}$ is negligible compared with total computational time for the discrete problem. The adaptive DtN method generates locally refined meshes of $\Omega$, while the adaptive PML method produces automatically coarse mesh size away from the outer boundary of $\Omega$.

Example 5.2. This example concerns the scattering of the plane wave $u_{I}=e^{\mathrm{i} k d \cdot x}$ with $\boldsymbol{d}=(1,0)$ by a U-shaped obstacle $D$ which is contained in the box

$$
\left\{x \in \Omega:-2.0<x_{1}<2.2,-0.7<x_{2}<0.7\right\} .
$$



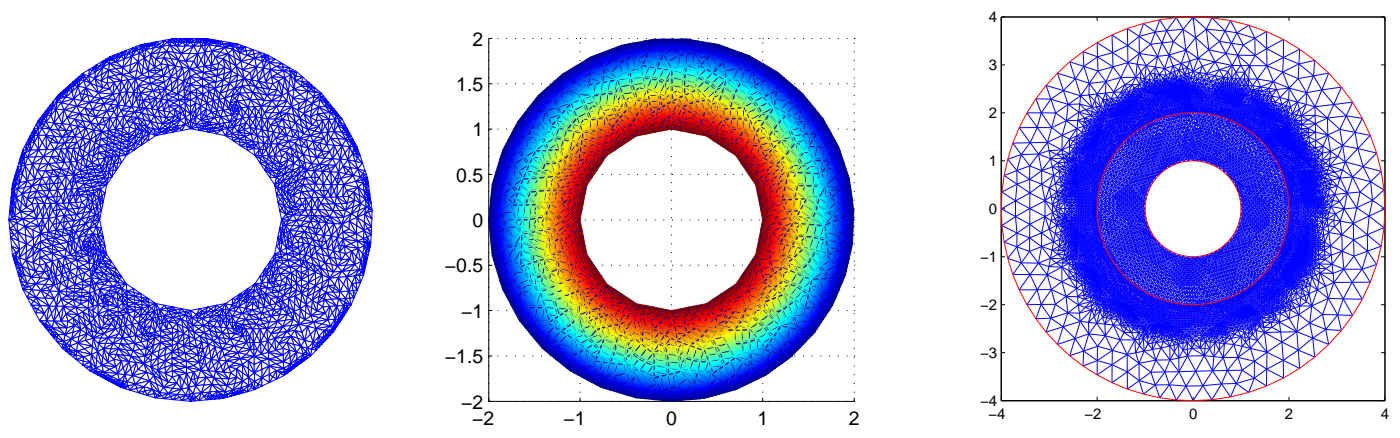

Figure 3: Example 5.1 with wavenumber $k=1$. Left: an adaptively refined mesh with 5223 elements using DtN; Right: the graph of $\left|u_{h}^{N}\right|$.
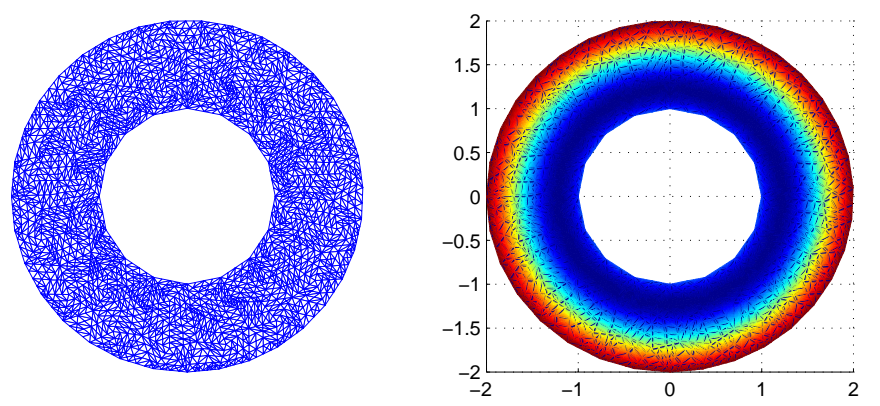

Figure 5: Example 5.1 with wavenumber $k=\pi$. Left: an adaptively refined mesh with 4680 elements using DtN; Right: the graph of $\left|u_{h}^{N}\right|$.
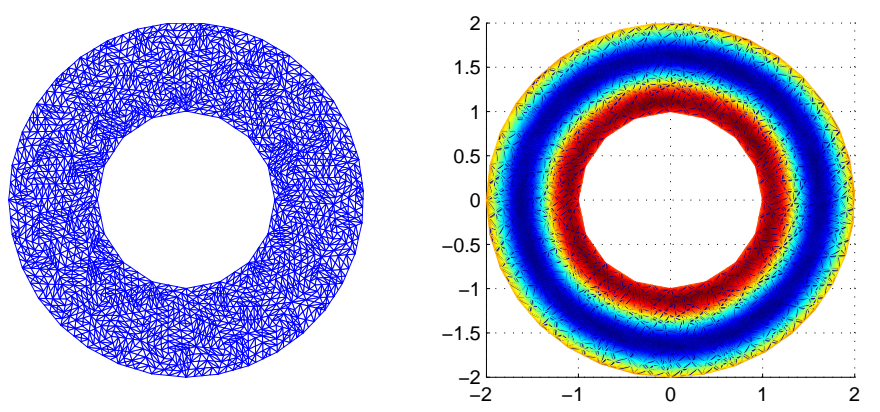

Figure 7: Example 5.1 with wavenumber $k=2 \pi$. Left: an adaptively refined mesh with 4336 elements using DtN; Right: the graph of $\left|u_{h}^{N}\right|$.

Figure 4: An adaptively refined mesh with 27,550 elements using the adaptive PML method for Example 5.1 with wavenumber $k=1$.

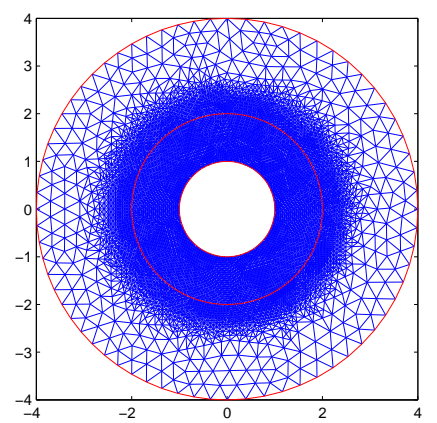

Figure 6: An adaptively refined mesh with 22,512 elements using the adaptive PML method for Example 5.1 with wavenumber $k=\pi$.

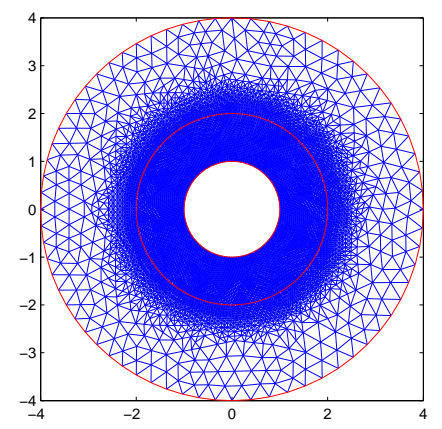

Figure 8: An adaptively refined mesh with 26,752 elements using the adaptive PML method for Example 5.1 with wavenumber $k=2 \pi$. 

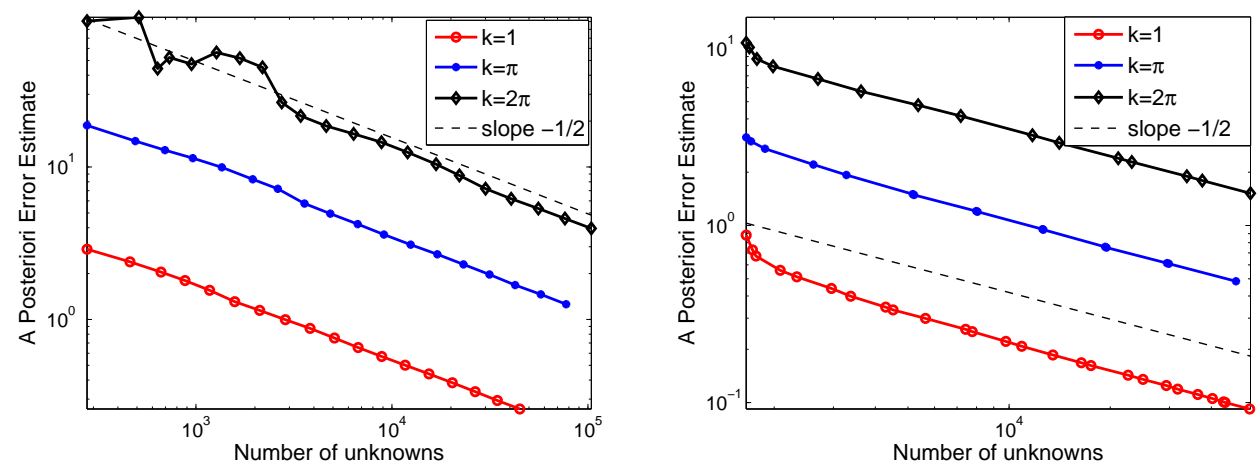

Figure 9: Quasi-optimality of the a priori error and a posteriori error estimate for Example 5.2. Left: the adaptive DtN method; Right: the adaptive PML method.
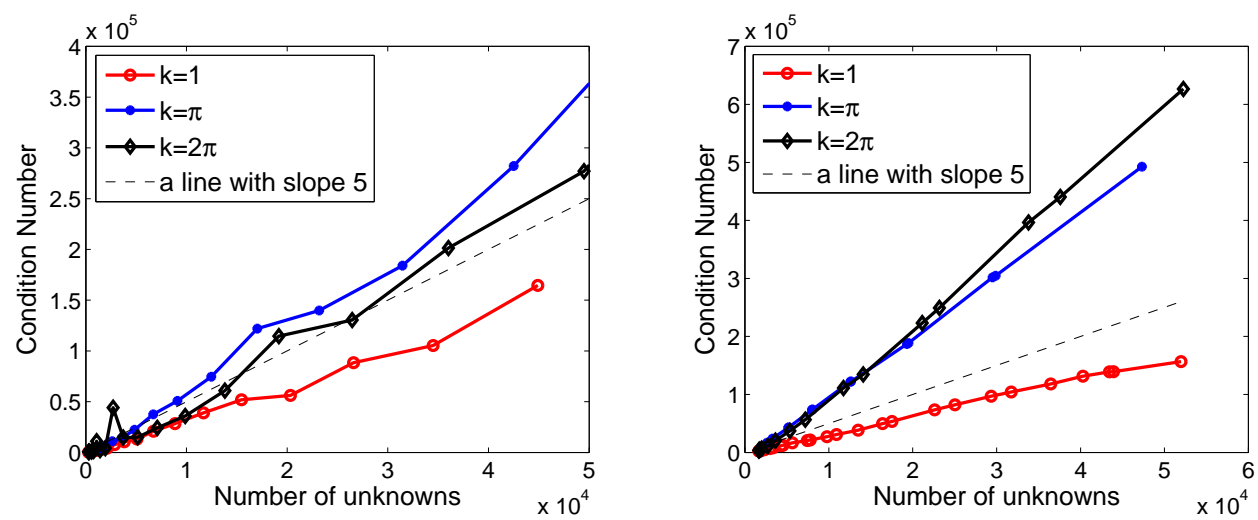

Figure 10: Condition number of the stiffness matrix for Example 5.2. Left: the adaptive DtN method; Right: the adaptive PML method.

The Dirichlet boundary condition is set by $g=-u_{I}$ on $\Gamma_{D}$. We define the truncation boundary by setting $R=3$. We set $N_{0}=3$ and $N_{\text {step }}=1$ for the adaptive DtN method and set the thickness of the PML by $\rho=3$ for the adaptive PML method.

Fig. 9 also shows that the quasi-optimality

$$
\left\|u-u_{h}\right\|_{1, \Omega} \sim C N_{h}^{-1 / 2}, \quad \bar{\eta}_{h} \sim C N_{h}^{-1 / 2} .
$$

hold asymptotically for both the adaptive DtN method and the adaptive PML method for $k=1, \pi, 2 \pi$. Fig. 10 shows the condition numbers of the finite element stiffness matrices for the adaptive DtN method and the adaptive PML method respectively. They are comparable in terms of approximation errors and condition numbers. Figs. 11-16 show the adaptively refined meshes and the plots of the discrete solution for different $k$. The adaptive DtN method generates locally refined meshes of $\Omega$ according to the variation of the discrete solutions, while the adaptive PML method produces automatically coarse mesh size away from the outer boundary of $\Omega$. 

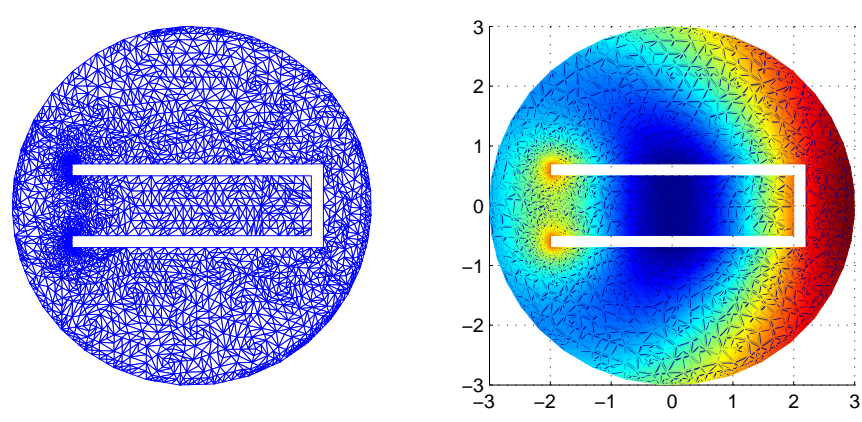

Figure 11: An adaptive mesh of 5381 elements after 8 iterations using DtN approach with $k=1$ for Example 5.2.
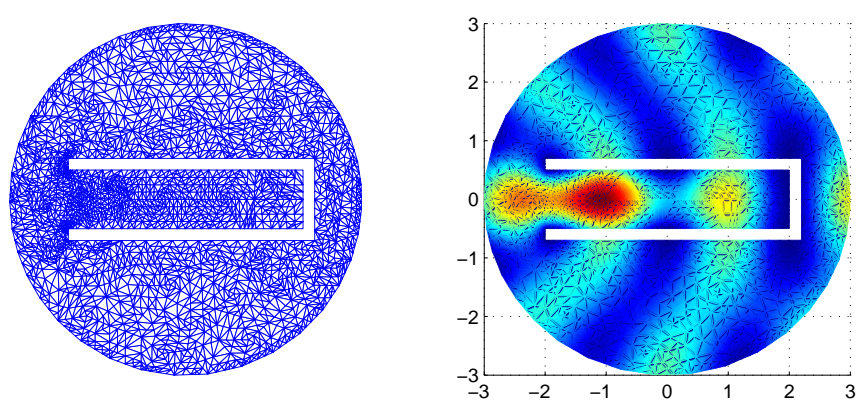

Figure 13: An adaptive mesh of 4933 elements after 7 iterations using DtN approach with $k=\pi$ for Example 5.2.
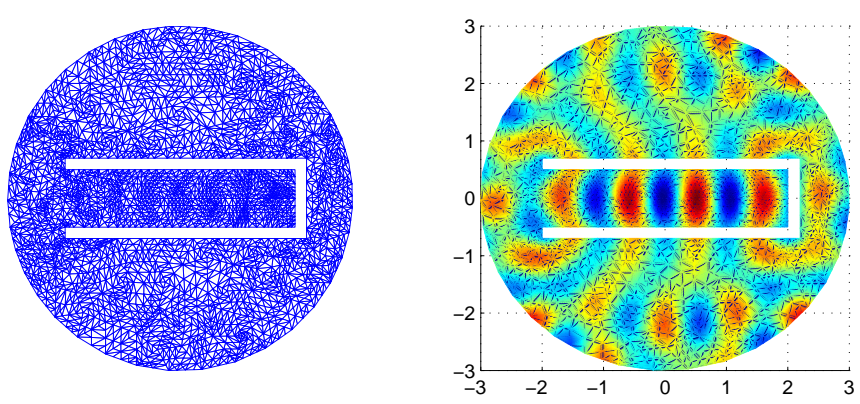

Figure 15: An adaptive mesh of 5995 elements after 9 iterations using DtN approach with $k=2 \pi$ for Example 5.2.

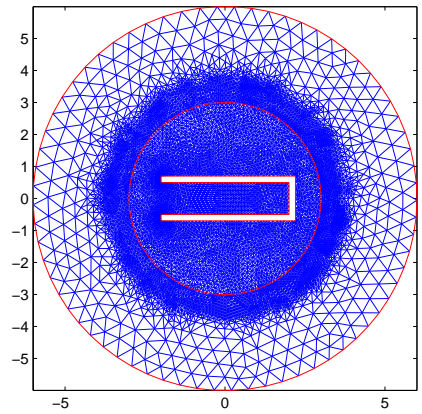

Figure 12: An adaptive mesh of 21,480 elements after 8 iterations using PML approach with $k=1$ for Example 5.2.

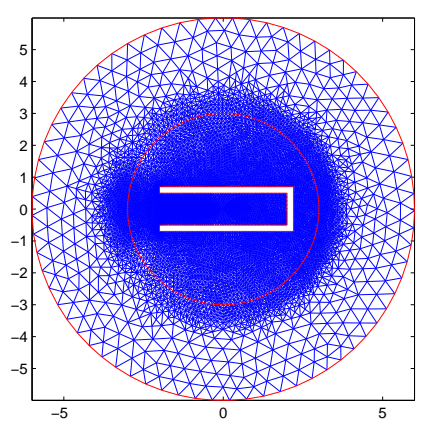

Figure 14: An adaptive mesh of 24,728 elements after 8 iterations using PML approach with $k=\pi$ for Example 5.2.

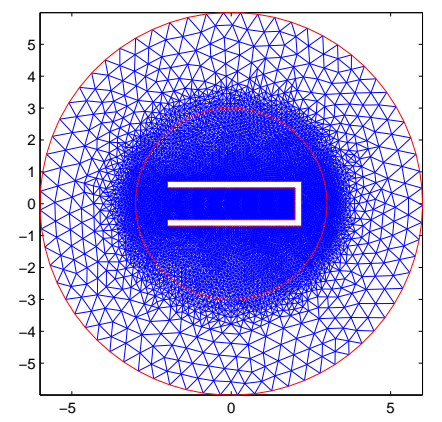

Figure 16: Adaptively refined meshes of 23,062 elements sing the adaptive PML method with $k=2 \pi$ for Example 5.2. 


\section{Concluding remarks}

We presented an adaptive finite element with DtN boundary condition for solving the acoustic wave scattering problem modeled by the two-dimensional Helmholtz equation. Based on the DtN operator, the transparent boundary condition is exact and the artificial boundary can be put as close as possible to the obstacle. Convergence analysis and a posterior error estimate was studied for the finite element solution. Numerical experiments were shown to compare with the performance by the adaptive PML finite element method. The proposed adaptive DtN finite element method is comparable with the adaptive PML finite element method in terms of convergence rate, condition numbers, and accuracy. The present work proposes the adaptive DtN method as a viable alternative for solving the two-dimensional obstacle scattering problem. We plan to employ the method in three-dimensional acoustic and electromagnetic wave scattering problems. We hope the adaptive DtN finite element method can be used to solve not only the scattering problems but also other scientific problems posed in unbounded domain, especially when the PML techniques might not be applicable. In conclusion then, our point of view is that the adaptive DtN finite element method enriches the range of choices available for solving the acoustic and electromagnetic scattering problems.

\section{Acknowledgments}

The research of PL was supported in part by the NSF grants DMS-0914595 and DMS1042958. The research of WZ was supported in part by China NSF under the grants 11031006 and 11171334, by the Funds for Creative Research Groups of China (Grant No. 11021101), and by the National Magnetic Confinement Fusion Science Program (Grant No. 2011GB105003).

\section{References}

[1] R. Adams, Sobolev Spaces, Academic Press, New York, 1975.

[2] I. Babuška and A. Aziz, Survey Lectures on Mathematical Foundations of the Finite Element Method, in The Mathematical Foundations of the Finite Element Method with Application to the Partial Differential Equations, ed. by A. Aziz, Academic Press, New York, 1973, 5-359.

[3] G. Bao, J. Li, P. Li, Z. Wang, and H. Wu, An adaptive finite element method with DtN boundary condition for the diffraction grating problem, preprint.

[4] Z. Chen and $\mathrm{H}$. Wu, An adaptive finite element method with perfectly matched absorbing layers for the wave scattering by periodic structures, SIAM J. Numer. Anal., 41 (2003), 799826.

[5] Z. Chen and X. Liu, An adaptive perfectly matched layer technique for time-harmonic scattering problems, SIAM J. Numer. Anal., 43 (2005), 645-671.

[6] D. Colton and R. Kress, Integral equation methods in scattering theory, John Wiley \& Sons, New York, 1983. 
[7] D. Colton and R. Kress, Inverse Acoustic and Electromagnetic Scattering Theory, Second Edition, Springer, Berlin, New York, 1998.

[8] B. Engquist and A. Majda, Absorbing boundary conditions for the numerical simulation of waves, Math. Comp., 31 (1977), 629-651.

[9] D. Gilbarg and N. Trudinger, Elliptic Partial Differential Equations of Second Order, Dordrecht: Kluwer, 1983.

[10] M. Grote and C. Kirsch, Dirichlet-to-Neumann boundary conditions for multiple scattering problems, J. Comput. Phys., 201 (2004), 630-650.

[11] T. Hagstrom, Radiation boundary conditions for the numerical simulation of waves, Acta Numerica (1999), 47-106.

[12] G.C. Hsiao, N. Nigamb, J.E. Pasciak, L. Xu, Error analysis of the DtN-FEM for the scattering problem in acoustics via Fourier analysis, J. Comput. Appl. Math., 235 (2011), 4949-4965.

[13] F. Ihlenburg and I. Babuška, Finite element solution of the Helmholtz equation with high wavenumber part I: the h-version of the FEM, Computers Math. Applic., 30 (1995), 9-37.

[14] F. Ihlenburg and I. Babušska, Finite element solution of the Helmholtz equation with high wavenumber part II: the hp-version of the FEM, SIAM J. Numer. Anal., 34 (1997), 315-358.

[15] D. Jerison and C. Kenig, Unique continuation and absence of positive eigenvalues for Schrödinger operators, Ann. Math., 121 (1985), 463-488.

[16] J. Jin, The Finite Element Method in Electromagnetics, New York: Wiley, 1993.

[17] R. Kress, Minimizing the condition number of boundary integral operators in acoustic and electromagnetic scattering, Q. Jl. Mech. Appl. Math., 38 (1985), 323-341.

[18] J. Li, An Adaptive Finite Element Method for 1D Diffraction Gratings, Nanjing University, Master Thesis, 2010.

[19] P. Monk, Finite Element Methods for Maxwell's Equations, Clarendon Press, Oxford, 2003.

[20] J.-C. Nédélec, Acoustic and Electromagnetic Equations Integral Representations for Harmonic Problems, Springer, 2000.

[21] D. Yu, The Natural Boundary Integral Method and Its Applications, Science Press \& Kluwer Academic Publishers, 2002. 